\title{
Space-Based Nonlinear Dynamic Inversion Control for Aircraft Continuous Descent Approach
}

\author{
Hakim Bouadi \\ MAIAA, Automation Research Group \& \\ Air Transportation Department \\ National Civil Aviation Institute \\ 07, Avenue Edouard Belin, 31400 Toulouse, France \\ Email: Hakim.Bouadi@enac.fr
}

Abstract-With the growth of civil aviation traffic, enhanced
accuracy performances are required from guidance systems to
maintain efficiency and safety in flight operations. This communi-
cation proposes a new representation of aircraft flight dynamics
at approach for landing and a space-based nonlinear dynamic
inversion control for a transportation aircraft. The main novelty
is that the adopted independent variable is the distance to land.
This new representation of flight dynamics should support the
development of improved aircraft guidance systems.

\section{INTRODUCTION}

With the growth of civil aviation traffic capacity, safety and environmental considerations urge today for the development of guidance systems with improved accuracy for spatial trajectory tracking. This should induce increased capacity by allowing safe operations at minimum seperation standards while trajectory dispersion resulting in extended noise impacts on surrounding communities should be reduced.

Current civil aviation guidance systems operate by real time corrective actions to maintain the aircraft trajectory as close as possible to the planned trajectory $[5,6]$. Wind is one of the main causes of guidance errors $[8,7,11]$. These guidance errors are detected by navigation systems whose accuracy have known large improvements with the hybridization of inertial units with sattelite information. However, until today vertical guidance remains problematic $[13,15]$ and covariance errors [12] are still large considering the time-based control laws which are applied $[9,11]$.

In this communication, we propose a representation of aircraft flight dynamics where the independent variable, the distance to land, is considered to be available on line. Based on a spatial representation of a transportation aircraft longitudinal flight dynamics, nonlinear dynamic inversion control is applied where the modelling of wind and wind effects on the flight dynamics is particularly considered.

Once the space based flight equations are established and a wind model is adopted, simulations are performed in the case of a continuous descent approach (CDA) where the guidance law allows to pursue a reference altitude for each distance to land as well as a reference airspeed. Simulation results display the effectiveness of the new approach.

\author{
F. Mora-Camino \\ MAIAA, Automation Research Group \& \\ Air Transportation Department \\ National Civil Aviation Institute \\ 07, Avenue Edouard Belin, 31400 Toulouse, France \\ Email: moracamino@hotmail.fr
}

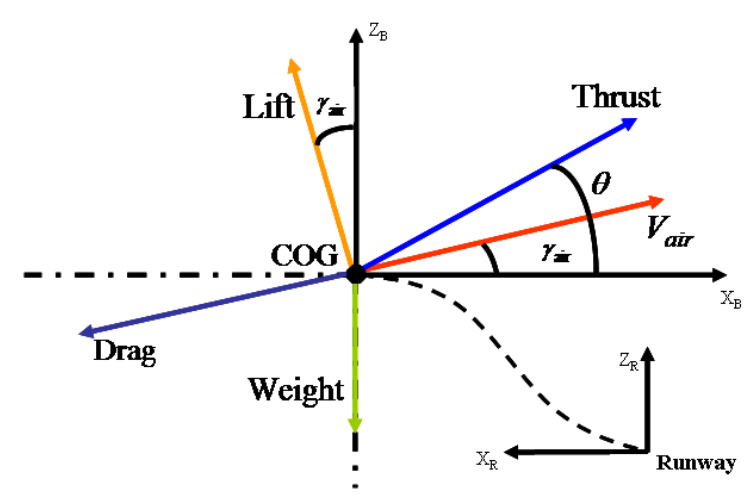

Fig. 1. Aircraft forces

\section{Space Referenced Vertical Guidance Dynamics}

The motion of an approach/descent transportation aircraft along a landing trajectory will be referenced with respect to a RRF (Runway Reference Frame) where its origin is located at the runway entrance as shown in Fig. 1.

The vertical plane components of the inertial speed are such as:

$$
\begin{gathered}
\dot{x}=-V_{a i r} \cos \gamma_{a i r}+w_{x} \\
\dot{z}=V_{a i r} \sin \gamma_{a i r}+w_{z}
\end{gathered}
$$

and inversely:

$$
\begin{gathered}
V_{a i r}=\sqrt{\left(\dot{x}-w_{x}\right)^{2}+\left(\dot{z}-w_{z}\right)^{2}} \\
\gamma_{a i r}=-\arctan \left(\frac{\dot{z}-w_{z}}{\dot{x}-w_{x}}\right)
\end{gathered}
$$

where $x$ and $z$ are the vertical plane coordinates of the aircraft center of gravity in the runway reference system, $V_{\text {air }}$ is the airspeed modulus, $\gamma_{a i r}$ is the airspeed path angle, $w_{x}$ and $w_{z}$ are the wind components in the RRF.

Adopting classical assumptions such as the RRF being an inertial frame, local flatness of the Earth, constant aircraft mass. The translational acceleration equations can be written 
as:

$$
\begin{gathered}
m \ddot{x}=- \\
+\cos \theta+D\left(z, V_{a i r}, \alpha\right) \cos \gamma_{a i r} \\
+L\left(z, V_{a i r}, \alpha\right) \sin \gamma_{a i r} \\
m \ddot{z}=T \sin \theta-D\left(z, V_{a i r}, \alpha\right) \sin \gamma_{a i r}-m g \\
+L\left(z, V_{a i r}, \alpha\right) \cos \gamma_{a i r}
\end{gathered}
$$

$T, D$ and $L$ are respectively the thrust, drag and lift forces. The lift and drag forces are given by:

$$
\begin{aligned}
L & =\frac{1}{2} \rho(z) V_{a i r}^{2} S C_{Z} \\
D & =\frac{1}{2} \rho(z) V_{a i r}^{2} S C_{X}
\end{aligned}
$$

where $\rho(z), S, C_{Z}$ and $C_{X}$ represent the air density with respect to the altitude, the wing surface area, the lift and drag coefficients, respectively.

$$
\begin{gathered}
C_{Z}=C_{Z_{0}}+C_{Z_{\alpha}} \alpha \\
C_{X}=C_{0}+C_{1} \alpha+C_{2} \alpha^{2}
\end{gathered}
$$

According to the polar model, the aerodynamic parameters $C_{0}, C_{1}$ and $C_{2}$ are such as:

$$
\begin{gathered}
C_{0}=C_{X_{0}}+k C_{Z_{0}}^{2} \\
C_{1}=2 k C_{Z_{0}} C_{Z_{\alpha}} \\
C_{2}=k C_{Z_{\alpha}}^{2}
\end{gathered}
$$

Assuming first order dynamics with time constant $\tau$ for the engines, we get between commanded thrust $T_{C}$ and effective thrust $T$ the following relation:

$$
\dot{T}=\frac{1}{\tau}\left(T_{C}-T\right)
$$

Under the above assumptions, the pitch rate is given by:

$$
\dot{\theta}=q
$$

Equations $(3 a)$ and $(3 b)$ can be rewritten in the aircraft airspeed frame such as:

$$
\begin{aligned}
& \dot{V}_{a i r}= \frac{1}{m}\left[T \cos \alpha-D\left(z, V_{a i r}, \alpha\right)-m g \sin \gamma_{a i r}\right. \\
&\left.+m\left(\dot{w}_{x} \cos \gamma_{a i r}-\dot{w}_{z} \sin \gamma_{a i r}\right)\right] \\
& \dot{\gamma}_{a i r}=\frac{1}{m V_{a i r}} {\left[T \sin \alpha+L\left(z, V_{a i r}, \alpha\right)-m g \cos \gamma_{a i r}\right.} \\
&\left.-m\left(\dot{w}_{x} \sin \gamma_{a i r}+\dot{w}_{z} \cos \gamma_{a i r}\right)\right]
\end{aligned}
$$

where $\alpha$ denotes the angle of attack with:

$$
\alpha=\theta-\gamma_{\text {air }}
$$

Considering that during an approach/descent without holding manoeuvers of an aircraft $x(t)$ is invertible and that the ground speed at position $x$ is given by:

$$
V_{G}=\dot{x}=-V_{a i r} \cos \gamma_{a i r}+w_{x}
$$

it is possible to express during these manoeuvers all the flight variables with respect to $x$ and its derivatives.

Here the following notation is adopted: $\frac{d^{k} *}{d x^{k}}=*^{[k]}$ and the guidance dynamics can be written as:

$$
\begin{gathered}
z^{[1]}=\frac{d z}{d x}=\frac{d z}{d t} \frac{d t}{d x}=\frac{V_{a i r} \sin \gamma_{a i r}+w_{z}}{V_{G}} \\
\theta^{[1]}=\frac{q}{V_{G}} \\
T_{\text {air }}^{[1]}=\frac{T_{C}-T}{\tau V_{G}} \\
\gamma_{\text {air }}^{[1]}=\frac{1}{m V_{G}}\left[T \cos \alpha-D\left(z, V_{a i r}, \alpha\right)-m g \sin \gamma_{a i r}\right. \\
\left.+m\left(\dot{w}_{x} \cos \gamma_{a i r}-\dot{w}_{z} \sin \gamma_{a i r}\right)\right] \\
\left.-m\left(\dot{w}_{x} \sin \gamma_{a i r}+\dot{w}_{z} \cos \gamma_{a i r}\right)\right]
\end{gathered}
$$

III. WIND MODELLING

Wind has a major role in aircraft guidance errors [1,2] and thus cannot be ignored in this study. Here a realistic wind model is introduced. It is considered that the wind components in the vertical plane of the runway are such as:

$$
\begin{aligned}
& w_{x}=W_{x}(x, z, t) \\
& w_{z}=W_{z}(x, z, t)
\end{aligned}
$$

then, it is possible to write:

$$
\dot{w}_{x}=W_{x x} \dot{x}+W_{x z} \dot{z}+W_{x t}
$$

with:

$$
W_{x x}=\frac{\partial W_{x}}{\partial x} \quad W_{x z}=\frac{\partial W_{x}}{\partial z} \quad W_{x t}=\frac{\partial W_{x}}{\partial t}
$$

and

$$
\dot{w}_{z}=W_{z x} \dot{x}+W_{z z} \dot{z}+W_{z t}
$$

with:

$$
W_{z x}=\frac{\partial W_{z}}{\partial x} \quad W_{z z}=\frac{\partial W_{z}}{\partial z} \quad W_{z t}=\frac{\partial W_{z}}{\partial t}
$$

In this study, for the aircraft longitudinal dynamics, the wind is considered as follows:

$$
\begin{gathered}
W_{x}(x, z, t)=W_{x}(z)+\delta_{x}\left(V_{a i r}, z, t\right) \\
W_{z}(x, z, t)=\delta_{z}\left(V_{a i r}, z, t\right)
\end{gathered}
$$

where $W_{x}(z)$ and $\delta_{x, z}\left(V_{a i r}, z, t\right)$ represent the deterministic and stochastic components of the considered wind, respectively.

The deterministic wind speed component is expressed as a logarithmic increasing and turning function of the altitude $z$ as shown below:

$$
\begin{gathered}
W_{x}(z)=W_{0}(z) \ln \left(\frac{z}{z_{0}}\right) \\
W_{0}(z)=W_{0}^{*} \cos \left(\omega z+\varphi_{0}\right)
\end{gathered}
$$


where $\omega$ and $W_{0}^{*}$ denote the circular frequency and the magnitude of the considered wind.

The stochastic wind component is given by its spectrum. A commonly used spectrum for wind turbulence modelling is the Dryden spectrum [4]. Then, considering that $\sigma_{x}$ and $\sigma_{z}$ are the turbulence standard deviations of independent processes and as an indicator for the atmospheric conditions it is possible to take the wind speed at $20 \mathrm{ft}$ above the ground $\left(W_{20}\right)$. For moderate conditions, $W_{20}=15.4 \mathrm{~m} / \mathrm{s}$ is selected. The turbulence standard deviation $\sigma_{z}$ is then given as follows:

$$
\sigma_{z}=0.1 W_{20}
$$

while $\sigma_{x}$ is assumed to be function of $\sigma_{z}$ and the altitude $z$.

- For $z \leq 305 \mathrm{~m}$ :

$$
\sigma_{x}=\frac{\sigma_{z}}{(0.177+0.0027 z)^{0.4}}
$$

- For $z>305 \mathrm{~m}$ :

$$
\sigma_{x}=\sigma_{z}
$$

The turbulence components can be generated from unitary white noise processes applied to the linear filters of the following transfer functions:

for $\delta_{x}\left(V_{a i r}, z, t\right)$ :

$$
H_{W_{x}}(s)=\sigma_{x} \sqrt{\frac{2 L_{x x}}{V_{a i r}}} \frac{1}{1+\frac{L_{x x}}{V_{a i r}} s}
$$

and for $\delta_{z}\left(V_{a i r}, z, t\right)$ :

$$
H_{W_{z}}(s)=\sigma_{z} \sqrt{\frac{L_{z z}}{V_{a i r}}} \frac{1+\sqrt{3} \frac{L_{z z}}{V_{a i r}} s}{\left(1+\frac{L_{z z}}{V_{a i r}} s\right)^{2}}
$$

where the turbulence lenghts $L_{x x}$ and $L_{z z}$ are function of the altitude:

- For $z \leq 305 \mathrm{~m}$ :

$$
\begin{gathered}
L_{x x}=\frac{z}{(0.177+0.0027 z)^{1.2}} \\
L_{z z}=z
\end{gathered}
$$

- For $z>305 \mathrm{~m}$ :

$$
L_{x x}=L_{z z}=305 m
$$

\section{Vertical Trajectory Tracking Control OBJECTIVES}

The independent inputs to the above flight dynamics are $q$, $T_{C}, w_{x}$ and $w_{z}$, where wind components $w_{x}$ and $w_{z}$ operate as external disturbances while $q$ is the result of pitch control and $T_{C}$ is the engine thrust setting.

Note that, the space-based state equation related to the innerloop pitch control is such as:

$$
q^{[1]}=\frac{d q}{d t} \frac{d t}{d x}=\frac{\dot{q}}{V_{G}}=\frac{M}{I_{y} V_{G}}
$$

where $M, I_{y}$ denote respectively the pitch moment and inertia moment according to the aircraft lateral axis where:

$$
M=\frac{1}{2} \rho(z) V_{a i r}^{2} S \bar{c}\left(C_{m_{0}}+C_{m_{\alpha}} \alpha+C_{m_{q}} \frac{q \bar{c}}{2 V_{a i r}}+C_{m_{\delta e}} \delta_{e}\right)
$$

with $\bar{c}$ and $\delta_{e}$ represent the aerodynamic mean chord line and elevator deflection, respectively.

Our main control objective is to make the aircraft follow with accuracy a trajectory $z_{d}(x)$ with $x \in\left[x_{0}, 0\right]$ while its airspeed is to remain in a safe domain by following a speed profile. Let $V_{a i r_{d}}(x)$ with $x \in\left[x_{0}, 0\right]$ be the desired airspeed profile.

Here $V_{a i r_{d}}(x)$ should be chosen to warrantee a sufficient margin with respect to the stall speed but also to provide a final ground speed compatible with the flare and landing manoeuvers. In addition, one of the main airworthiness requirements to be met is the maximum operating speed $V_{M O}$.

Let $V_{r}(x)$ be the reference ground speed profile. The desired airspeed profile is chosen to guarantee:

- For low speeds, a minimum margin with respect to the stall speed:

$$
V_{a i r_{d}}(x)=\operatorname{Max}\left\{V_{S}\left(z_{d}(x)\right)+\Delta V_{\text {min }}, V_{r}(x)-\hat{w}_{x}(x)\right\}
$$

where $V_{S}, \Delta V_{\text {min }}$ and $\hat{w}_{x}$ are the stall speed, the minimum margin speed and the estimate of the horizontal wind speed, respectively.

- For high speeds, an airspeed less than the maximum operating speed:

$$
V_{a i r_{d}}(x)=\operatorname{Min}\left\{V_{M O}\left(z_{d}(x)\right), V_{r}(x)-\hat{w}_{x}(x)\right\}
$$

- In all other cases:

$$
V_{a i r_{d}}(x)=V_{r}(x)-\hat{w}_{x}(x)
$$

\section{SPACE-BASEd NLI TRACKING CONTROL}

In this section a nonlinear inverse control technique will be applied to the spatial dynamics of the aircraft which will be such as its tracking errors follow decoupled linear spatial dynamics such as:

$$
\sum_{k=0}^{K} a_{k}\left(y^{[k]}-y_{d}^{[k]}\right)=0
$$

where $y$ is either $z(x)$ or $V_{a i r}(x)$ and $y_{d}$ is the corresponding desired profile. $[k]$ denotes the $k^{\text {th }}$ space derivative of the considered output $y(x)$ and $(K-1)$ is equal to the relative degree [14] of the output $y$.

The trajectory output variables equations can be written 
under an affine form with respect to the inputs $q$ and $T_{C}$ :

$$
\begin{aligned}
& V_{a i r}^{[2]}=\frac{1}{V_{G}^{2}}\left[A_{V}\left(z, \alpha, V_{a i r}, T, W\right)+B_{V_{q}}\left(z, \alpha, V_{a i r}, T, W\right) q\right. \\
& \left.+B_{V_{T}}\left(z, \alpha, V_{a i r}, T, W\right) T_{C}\right] \\
& z^{[3]}=\frac{1}{V_{G}^{2}}\left[A_{z}\left(z, \alpha, V_{a i r}, T, W\right)+B_{z_{q}}\left(z, \alpha, V_{a i r}, T, W\right) q\right. \\
& \left.+B_{z_{T}}\left(z, \alpha, V_{a i r}, T, W\right) T_{C}\right]
\end{aligned}
$$

where $W$ represent the parameters $w_{x}, w_{z}, \dot{w}_{x}, \dot{w}_{z}$ and $\ddot{w}_{x}$, $\ddot{w}_{z}$ which can be expressed successively according to (14) and (16).

The components $A_{V}, B_{V_{q}}, B_{V_{T}}$ and $A_{z}, B_{z_{q}}, B_{z_{T}}$ are given by:

$$
\begin{aligned}
A_{V}= & \frac{1}{m}\left[-\frac{T}{\tau} \cos \alpha+T \dot{\gamma}_{a i r} \sin \alpha-\rho(z) V_{a i r} \dot{V}_{a i r} S C_{X}\right. \\
& +\frac{1}{2} \rho(z) V_{a i r}^{3} S\left(C_{1} \dot{\gamma}_{a i r}+2 C_{2} \dot{\gamma}_{a i r} \alpha\right)+W_{x x}\left(\ddot{x} \cos \gamma_{a i r}\right. \\
& \left.-\dot{x} \dot{\gamma}_{a i r} \sin \gamma_{a i r}\right)+W_{x z}\left(\ddot{z} \cos \gamma_{a i r}-\dot{z} \dot{\gamma}_{a i r} \sin \gamma_{a i r}\right) \\
& -W_{z x}\left(\ddot{x} \sin \gamma_{a i r}+\dot{x}_{a i r} \cos \gamma_{a i r}\right)-W_{z z}\left(\ddot{z} \sin \gamma_{a i r}\right. \\
& \left.+\dot{z}_{a i r} \cos \gamma_{a i r}\right)+\dot{W}_{x t} \cos \gamma_{a i r}-W_{x t} \dot{\gamma}_{a i r} \sin \gamma_{a i r} \\
& -\dot{W}_{z t} \sin \gamma_{a i r}-W_{z t} \dot{\gamma}_{a i r} \cos \gamma_{a i r}-\frac{\dot{V}_{a i r}}{V_{G}} \\
& \times\left(-\dot{V}_{a i r} \cos \gamma_{a i r}+V_{a i r} \dot{\gamma}_{a i r} \sin \gamma_{a i r}+W_{x x} \dot{x}\right. \\
& \left.\left.+W_{x z} \dot{z}+W_{x t}\right)-m g \dot{\gamma}_{a i r} \cos \gamma_{a i r}\right] \\
B_{V_{q}} & =\frac{1}{m}\left[-T \sin \alpha-\frac{1}{2} \rho(z) V_{a i r}^{2} S C_{1}-\rho(z) V_{a i r}^{2} S C_{2} \alpha\right]
\end{aligned}
$$

and

$$
\begin{aligned}
A_{z}= & \frac{1}{V_{G}^{2}}\left[A_{V}\left(w_{x} \sin \gamma_{a i r}+w_{z} \cos \gamma_{a i r}\right)+F\left(z, \alpha, V_{a i r}, T, W\right)\right. \\
& \times\left\{-V_{a i r}^{2}+V_{a i r}\left(w_{x} \cos \gamma_{a i r}-w_{z} \sin \gamma_{a i r}\right)\right\} \\
& \left.+\Upsilon\left(z, \alpha, V_{\text {air }}, T, W\right) V_{G}^{2}\right]
\end{aligned}
$$

with $\Upsilon\left(z, \alpha, V_{a i r}, T, W\right)$ and $F\left(z, \alpha, V_{a i r}, T, W\right)$ are such as:

$$
\begin{aligned}
\Upsilon= & \frac{1}{V_{G}^{2}}\left[-2 V_{a i r} \dot{V}_{a i r} \dot{\gamma}_{a i r}+2 \dot{V}_{a i r} \dot{\gamma}_{a i r}\left(w_{x} \cos \gamma_{a i r}-w_{z} \sin \gamma_{a i r}\right)\right. \\
& -V_{a i r} \dot{\gamma}_{a i r}^{2}\left(w_{x} \sin \gamma_{a i r}+w_{z} \cos \gamma_{a i r}\right)-V_{a i r}\left(\ddot{w}_{z} \cos \gamma_{a i r}\right. \\
& \left.+\ddot{w}_{x} \sin \gamma_{a i r}\right)+w_{x}\left(W_{z x} \ddot{x}+W_{z z} \ddot{z}+\dot{W}_{z t}\right)-w_{z}\left(W_{x x} \ddot{x}\right. \\
& \left.+W_{x z} \ddot{z}+\dot{W}_{x t}\right)-\frac{2}{V_{G}}\left(-\dot{V}_{a i r} \cos \gamma_{a i r}+V_{a i r} \dot{\gamma}_{a i r} \sin \gamma_{a i r}\right. \\
& \left.+\dot{w}_{x}\right) \times\left\{-V_{a i r}^{2} \dot{\gamma}_{a i r}-V_{a i r}\left(\dot{w}_{z} \cos \gamma_{a i r}+\dot{w}_{x} \sin \gamma_{a i r}\right)\right. \\
& +V_{a i r} \dot{\gamma}_{a i r} \times\left(w_{x} \cos \gamma_{a i r}+w_{z} \sin \gamma_{a i r}\right)+\dot{V}_{a i r}\left(w_{x} \sin \gamma_{a i r}\right. \\
& \left.-w_{z} \cos \gamma_{a i r}\right)+w_{x}\left(W_{z x} \dot{x}+W_{z z} \dot{z}+W_{z t}\right)+w_{z}\left(W_{x x} \dot{x}\right. \\
& \left.\left.\left.+W_{x z} \dot{z}+W_{x t}\right)\right\}\right]
\end{aligned}
$$

$$
\begin{aligned}
F= & \frac{1}{m V_{a i r}}\left[-\frac{T}{\tau} \sin \alpha-T \dot{\gamma}_{a i r} \cos \alpha+\rho(z) V_{a i r} \dot{V}_{a i r} S C_{Z}\right. \\
& -\frac{1}{2} \rho(z) V_{a i r}^{2} S C_{Z_{\alpha}} \dot{\gamma}_{a i r}+m g \dot{\gamma}_{a i r} \sin \gamma_{a i r}-m\left(\ddot{w}_{x} \sin \gamma_{a i r}\right. \\
& \left.+\dot{w}_{x} \dot{\gamma}_{a i r} \cos \gamma_{a i r}+\ddot{w}_{z} \cos \gamma_{a i r}-\dot{w}_{x} \dot{\gamma}_{a i r} \sin \gamma_{a i r}\right) \\
& -\frac{m \dot{\gamma}_{a i r}}{V_{G}}\left\{-V_{a i r}^{2} \dot{\gamma}_{a i r} \sin \gamma_{a i r}+\dot{V}_{a i r} w_{x}-V_{a i r}\left(W_{x x} \dot{x}\right.\right. \\
& \left.\left.\left.+W_{x z} \dot{z}+W_{x t}\right)\right\}\right]
\end{aligned}
$$

and

$$
\begin{aligned}
B_{z_{q}}= & \frac{1}{V_{G}^{2}}\left[\frac{1}{m}\left(w_{x} \sin \gamma_{a i r}+w_{z} \cos \gamma_{a i r}\right) \times(-T \sin \alpha\right. \\
& \left.-\frac{1}{2} \rho(z) V_{a i r}^{2} S C_{1}-\rho(z) V_{a i r}^{2} S C_{2} \alpha\right)+\frac{1}{m V_{a i r}} \\
& \times\left\{-V_{a i r}^{2}+V_{a i r}\left(w_{x} \cos \gamma_{a i r}-w_{z} \sin \gamma_{a i r}\right)\right\} \\
& \left.\times\left(T \cos \alpha+\frac{1}{2} \rho(z) V_{a i r}^{2} S C_{Z_{\alpha}}\right)\right] \\
B_{z_{T}}= & \frac{1}{V_{G}^{2}}\left[\frac{\cos \alpha}{m \tau}\left(w_{x} \sin \gamma_{a i r}+w_{z} \cos \gamma_{a i r}\right)+\frac{\sin \alpha}{m V_{a i r} \tau}\right. \\
& \left.\times\left\{-V_{a i r}^{2}+V_{a i r}\left(w_{x} \cos \gamma_{a i r}-w_{z} \sin \gamma_{a i r}\right)\right\}\right]
\end{aligned}
$$

In the above equations $\dot{u}$ and $\ddot{u}$ with $u \in$ $\left\{x, z, \gamma_{a i r}, V_{a i r}, w_{x}, w_{z}\right\}$ denote quantities:

$$
\begin{gathered}
\dot{u}=u^{[1]} V_{G} \\
\ddot{u}=u^{[2]} V_{G}{ }^{2}+u^{[1]} V_{G}^{[1]} V_{G}
\end{gathered}
$$

Now, it can be shown that in standard flight conditions matrix $\Psi$ given by:

$$
\Psi=\left(\begin{array}{cc}
B_{z_{q}} & B_{z_{T}} \\
B_{V_{q}} & B_{V_{T}}
\end{array}\right)
$$


is invertible. This property allows through dynamic inversion to get control law to perform trajectory tracking with exponential convergence $[3,14]$.

The desired vertical trajectory $z_{d}(x)$ is expressed according to some constraints as a smooth polynomial function of the distance to touchdown $x$ :

$$
z_{d}(x)=\sum_{i=1}^{n} a_{i-1} x^{i-1}
$$

where the $a_{i}$ are real valued parameters.

Now, let $\xi_{z}(x)$ and $\xi_{\text {V air }}(x)$ be the tracking errors related to the desired altitude $z_{d}(x)$ and desired airspeed profile $V_{d}^{\text {air }}(x)$, respectively:

$$
\begin{gathered}
\xi_{z}(x)=z(x)-z_{d}(x) \\
\xi_{V_{a i r}}(x)=V_{a i r}(x)-V_{a i r_{d}}(x)
\end{gathered}
$$

Then, based on the nonlinear dynamic inversion technique, the control laws $q$ and $T_{C}$ are expressed in the space frame such as:

$$
\left(\begin{array}{c}
q \\
T_{C}
\end{array}\right)=\left(\begin{array}{cc}
B_{z_{q}} & B_{z_{T}} \\
B_{V_{q}} & B_{V_{T}}
\end{array}\right)^{-1}\left(\begin{array}{c}
V_{G}^{2} D_{z}(x)-A_{z} \\
V_{G}^{2} D_{V_{a i r}}(x)-A_{V}
\end{array}\right)
$$

with:

$$
\begin{aligned}
& D_{V_{a i r}}(x)=V_{a i r_{d}}^{[2]}(x)+k_{1 v} \xi_{V_{\text {air }}^{[1]}}^{[1}(x)+k_{2 v} \xi_{V_{\text {air }}}(x) \\
& D_{z}(x)=z_{d}^{[3]}(x)+k_{1 z} \xi_{z}^{[2]}(x)+k_{2 z} \xi_{z}^{[1]}(x)+k_{3 z} \xi_{z}(x)
\end{aligned}
$$

where $k_{1 v}, k_{2 v}, k_{1 z}, k_{2 z}$ and $k_{3 z}$ are real parameters such as the roots of $p^{2}+k_{1 v} p+k_{2 v}$ and $p^{3}+k_{1 z} p^{2}+k_{2 z} p+k_{3 z}$ produce adequate tracking error dynamics (convergence without oscillation over a given space segment) where $p$ denotes the Laplace variable.

\section{Simulation Study}

The control laws synthesis is completed by the following rate limits and saturations for the elevator deflection $\delta_{e}$ and the throttle setting $T_{C}$, respectively [4]:

$$
\begin{aligned}
-15 \frac{\pi}{180} \mathrm{rad} / \mathrm{s} & \leqslant \dot{\delta}_{e} \leqslant 15 \frac{\pi}{180} \mathrm{rad} / \mathrm{s} \\
-25 \frac{\pi}{180} \mathrm{rad} & \leqslant \delta_{e} \leqslant 10 \frac{\pi}{180} \mathrm{rad} \\
-1.6 \frac{\pi}{180} \mathrm{rad} / \mathrm{s} & \leqslant \dot{T}_{C} \leqslant 1.6 \frac{\pi}{180} \mathrm{rad} / \mathrm{s} \\
0.5 \frac{\pi}{180} \mathrm{rad} & \leqslant T_{C} \leqslant 10 \frac{\pi}{180} \mathrm{rad}
\end{aligned}
$$

while the minimum allowable speed is $1.23 \times V_{\text {stall }}$ with $V_{\text {stall }}=51.8 \mathrm{~m} / \mathrm{s}$ and the angle of attack is limited to the interval $\left[-11.5^{\circ}, 18^{\circ}\right]$ where $\alpha_{\text {stall }}=18^{\circ}$.

The previous modelling approach is applied to the Research Civil Aircraft Model (RCAM) which has the characteristics of a wide body transportation aircraft [4]. The chosen reference trajectory is a CDA (Continuous Descent Approach) profile which is known to reduce noise nuisance and fuel consumption. The main constraint is that the desired altitude trajectory joins the ILS (Instrument Landing System) glide
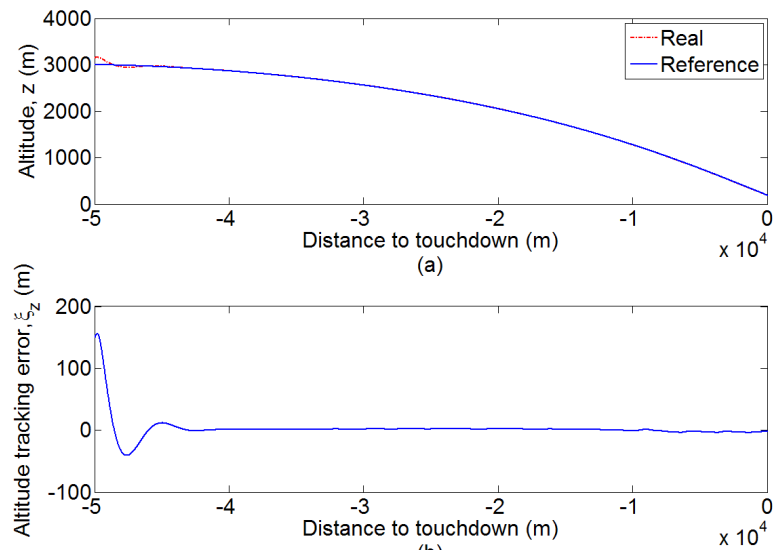

(b)

Fig. 2. Desired altitude tracking and altitude tracking error, respectively
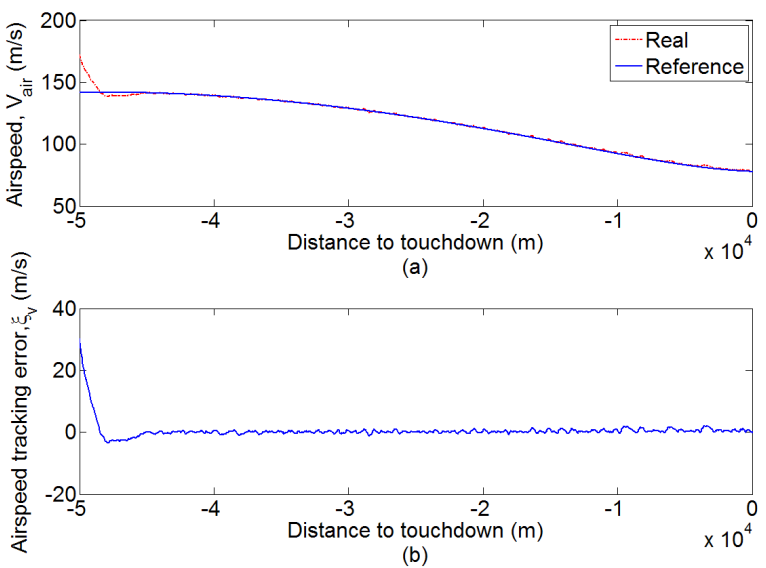

Fig. 3. Desired airspeed tracking and airspeed tracking error, respectively

path at $1000 \mathrm{~m}$ with an airspeed equal to $85 \mathrm{~m} / \mathrm{s}$ and a glide slope equal to $-3^{\circ}$.

Figure. 2 displays the tracking performance with respect to altitude. There the average guidance error remains quite constant around a mean value of $0.3 \mathrm{~m}$ even close to the touchdown point. The desired airspeed profile, is such that at $z_{d}(x)=3000 \mathrm{~m}$, the desired airspeed $V_{d}^{a i r}(x)$ is equal to $140 \mathrm{~m} / \mathrm{s}$ and at the touchdown point $V_{d}^{\text {air }}(x)$ is equal to $80 \mathrm{~m}$. Simulation results shown in Fig. 3 display a good tracking performance of desired airspeed profile. Figure. 4 displays respectively the resulting elevator deflection $\delta_{e}$ and the throttle setting $T_{C}$ where the Fig. 5 shows the wind components history.

\section{CONCLUSION}

In this communication a new representation of aircraft flight dynamics has been proposed. The main objective here is to promote space accuracy in trajectory tracking since this representation allows the development of new nonlinear guidance control laws. 


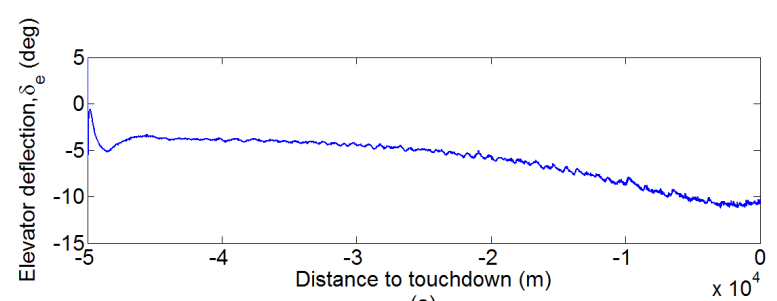

(a)

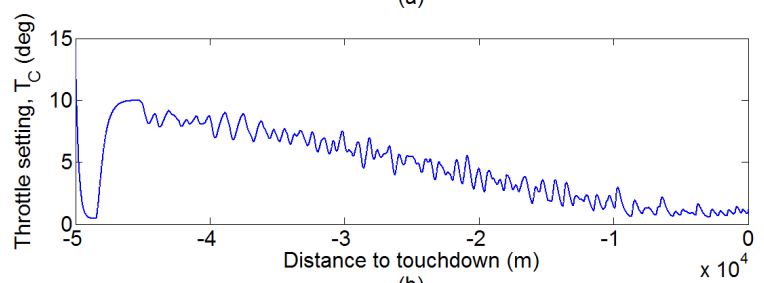

(b)

Fig. 4. Control inputs

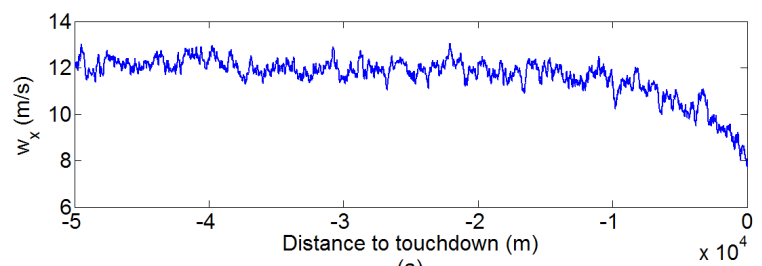

(a)

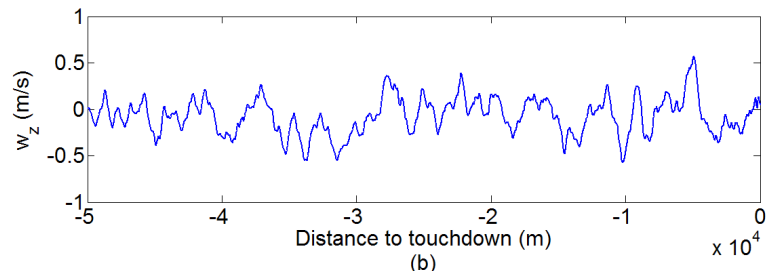

(b)

Fig. 5. Wind history
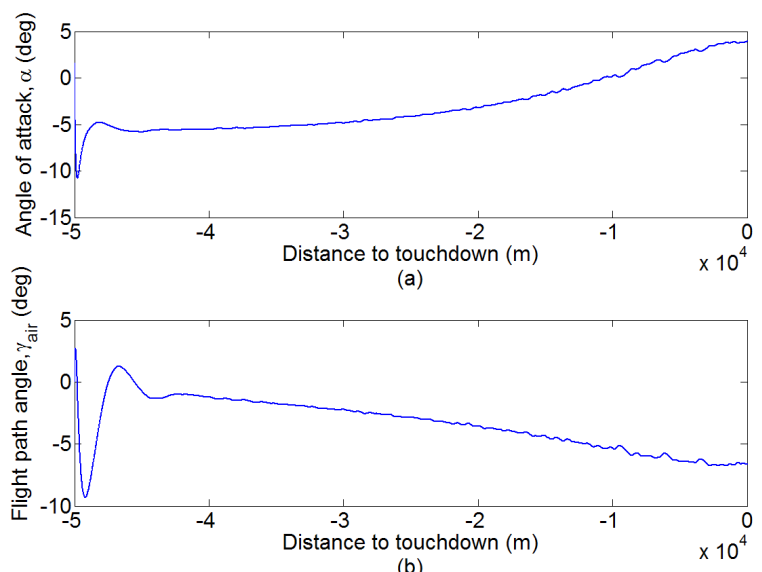

(b)

Fig. 6. Evolution of angle of attack and flight path angle, respectively

Then optimal trajectories with respect to fuel, or time of landing, or both of them computed by the FMS (Flight Management System) will be performed more accurately.

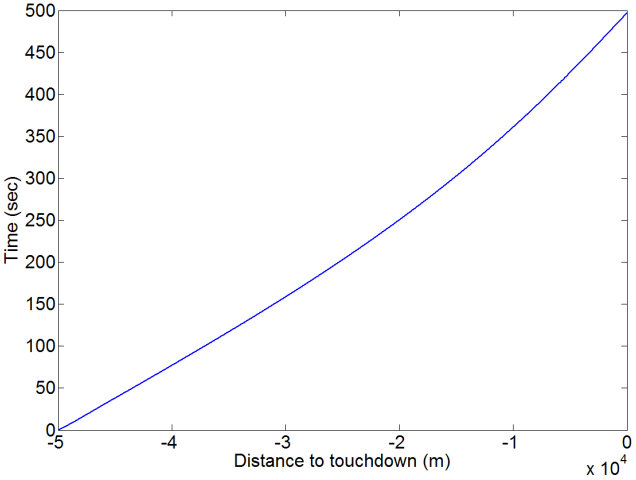

Fig. 7. Overfly time Vs. Space

Also, spatial dispersion will be diminuished with direct effects on safe separation between controlled aircraft (ATC) and on noise impact for airport surrounding population.

It appears also that many other types of vehicles have dynamics which, when written with respect to space, present the dynamic inversion property. So this approach can be also useful for other vehicles where accurate trajectory tracking is essential for their operations.

\section{REFERENCES}

[1] Etkin B, Dynamics of Atmospheric Flight, John Wiley and Sons, Inc, New York, 1985

[2] Frost W. and Bowles. R, Wind Shear Terms in the Equations of Aircraft Motion, Journal of Aircraft, Vol. 21, No.11, p. 866-872, 1984.

[3] Isidori A, " Nonlinear Control Systems", Springer-Verlag, Berlin.

[4] Magni J-F. et al, "Robust Flight Control, A Design Challenge", SpringerVerlag, London.

[5] Miele A, et al, "Guidance Strategies for Near-Optimum Takeoff Performance in Wind Shear", Journal of Optimization Theory and Applications, Vol. 50, No. 1, 1986.

[6] Miele A, et al, "Optimization and Gamma/Theta Guidance of Flight Trajectories in a Windshear", Presented at the 15th ICAS Congress, London, 1986.

[7] Miele A, et al, "Optimal Trajectories and Guidance Trajectories for Aircraft Flight Through Windshears", Proceedings of the 29th Conference on Decision and Control, Honolulu, Hawaii, pp. 737-746, 1990.

[8] Psiaki M.L. and R.F. Stengel, "Analysis of Aircraft Control Strategies for Microburst Encounter", Journal of Guidance, Control, and Dynamics, Vol. 8, No. 5, p. 553-559, 1985.

[9] Psiaki M.L, " Control of Flight Through Microburst Wind Shear Using Deterministic Trajectory Optimization", Ph.D. Thesis, Department of Mechanical and Aerospace Engineering, Princeton University, Report No. 1787-T, 1987.

[10] Psiaki M.L. and R.F. Stengel, "Optimal Aircraft Performance During Microburst Encounter", Journal of Guidance, Control, and Dynamics, Vol. 14, No. 2, p. 440-446, 1991.

[11] Psiaki M.L. and K. Park, "Thrust Laws for Microburst Wind Shear Penetration", Journal of Guidance, Control, and Dynamics, Vol. 15, No. 4, 1992.

[12] Sandeep S. Mulgund and Robert F. Stengel, " Optimal Nonlinear Estimation for Aircraft Flight Control in Wind Shear, Automatica, Vol. 32, No. 1, January 1996.

[13] Singh S.N. and W.J. Rugh, 'Decoupling in a Class of Nonlinear Systems by State Feedback", ASME Journal of Dynanmic Systems, Measurement, and Control, Series G, Vol. 94, p. 323-329, 1972.

[14] I.J.-J. Slotine and L. Weiping, Applied Nonlinear Control, Prentice Hall, Upper Saddle River, NJ; 1991.

[15] Stengel, R.F, "Toward Intelligent Flight Control", IEEE Trans. on Systems, Man, and Cybernetics, Vol. 23, No. 6, p. 1699-1717, 1993. 